\title{
Tate-Shafarevich Groups and Frobenius Fields of Reductions of Elliptic Curves
}

\author{
IGOR E. SHPARLINSKI \\ Dept. of Computing, Macquarie University \\ Sydney, NSW 2109, Australia \\ igor@ics.mq.edu.au
}

December 1, 2018

\begin{abstract}
Let $\mathbf{E} / \mathbb{Q}$ be a fixed elliptic curve over $\mathbb{Q}$ which does not have complex multiplication. Assuming the Generalized Riemann Hypothesis, A. C. Cojocaru and W. Duke have obtained an asymptotic formula for the number of primes $p \leq x$ such that the reduction of $\mathbf{E}$ modulo $p$ has a trivial Tate-Shafarevich group. Recent results of A. C. Cojocaru and C. David lead to a better error term. We introduce a new argument in the scheme of the proof which gives further improvement.
\end{abstract}

2000 Mathematics Subject Classification: 11G07, 11N35, 11L40, $14 \mathrm{H} 52$

\section{Introduction}

Let $\mathbf{E} / \mathbb{Q}$ be a fixed elliptic curve over $\mathbb{Q}$ of conductor $N$, we refer to $[7$ for the background on elliptic curves. For a prime $p \nmid N$ we denote the reduction of $\mathbf{E}$ modulo $p$ as $\mathbf{E}_{p} / \mathbb{F}_{p}$

As in [2], we use $\mathrm{III}_{p}$ to denote the Tate-Shafarevich group of $\mathbf{E}_{p} / \mathbb{F}_{p}$ which is an analogue of the classical Tate-Shafarevich group (see [7]) defined with 
respect to $\mathbf{E}_{p}$ and the function field $\mathbb{K}$ of $\mathbf{E}_{p}$, that is,

$$
\mathrm{III}_{p}=\operatorname{III}\left(\mathbf{E}_{p} / K\right)
$$

we refer to [2] for a precise definition.

Let $\pi_{T S}(x)$ be the counting function of primes $p \nmid N$ for which $\operatorname{III}_{p}$ is trivial. More formally,

$$
\pi_{T S}(x)=\#\left\{p \leq x \mid p \nmid N, \# \mathrm{III}_{p}=1\right\} .
$$

As usual, we also use $\pi(x)$ to denote the number of primes $p \leq x$.

Cojocaru and Duke [2, Proposition 5.3] have proved that if $\mathbf{E}$ does not have complex multiplication then under the Generalized Riemann Hypothesis $(\mathrm{GRH})$ the following asymptotic formula

$$
\pi_{T S}(x)=\alpha \pi(x)+R(x)
$$

holds for some explicitly defined constant $\alpha$ depending on $\mathbf{E}$, where

$$
R(x)=O\left(x^{53 / 54+o(1)}\right)
$$

(hereafter implicit constants in the symbols ' $O$ ', ' $\ll$ ' and ' $\gg$ ' may depend on $\mathbf{E}$ ). Furthermore, we have $\alpha>0$ if and only if $\mathbf{E}$ has an irrational point of order two.

The proof of (2) is based on the square sieve of Heath-Brown [4] combined with a bound of certain character sums. This character sum has been estimated in a sharper way by Cojocaru and David [1, Theorem 3], who also noticed that using their estimate in the proof of (2) from [2] reduces the error term in (1) to

$$
R(x)=O\left(x^{41 / 42+o(1)}\right) .
$$

Here we introduce some additional element in the approach of [2], which we also combine with the aforementioned stronger bound of character sums of [1, Theorem 3], to obtain a further improvement of (2) and (3). Namely, we obtain an extra saving from taking advantage of averaging over a certain parameter $m$, which appears in the argument of Cojocaru and Duke [2]. To take the most out of this, we apply the bound of double character sums due to Heath-Brown [5]. This yields the following estimate:

Theorem 1. Suppose $\mathbf{E}$ does not have complex multiplication and also assume that the GRH holds. Then the asymptotic formula (1) holds with

$$
R(x)=O\left(x^{39 / 40+o(1)}\right) .
$$


The main goal of [1] is to estimate $\Pi(\mathbb{K}, x)$ which is the number of primes $p \leq x$ with $p \nmid N$ and such that a root of the Frobenius endomorphism of $\mathbf{E}_{p} / \mathbb{F}_{p}$ generates the imaginary quadratic field $\mathbb{K}$. The famous Lang-Trotter conjecture, which asserts that if $\mathbf{E}$ does not have complex multiplication then

$$
\Pi(\mathbb{K}, x) \sim \beta(\mathbb{K}) \frac{x^{1 / 2}}{\log x}
$$

with some constant $\beta>0$ depending on $\mathbb{K}$ (and on $\mathbf{E}$ ), remains open. However, under the GRH, the bound

$$
\Pi(\mathbb{K}, x) \leq C(\mathbb{K}) \frac{x^{4 / 5}}{\log x} .
$$

has been given by Cojocaru and David [1, Theorem 2], where the constant $C(\mathbb{K})$ depends on $\mathbb{K}$ (and on $\mathbf{E}$ ). Moreover, using the aforementioned new bound of character sums, Cojocaru and David [1, Corollary 4] have given a weaker, but uniform with respect to $\mathbb{K}$, bound

$$
\Pi(\mathbb{K}, x)=O\left(x^{13 / 14} \log x\right) .
$$

For real $4 x \geq u>v \geq 1$, we now consider the average value

$$
\sigma(x ; u, v)=\sum_{\substack{u-v \leq m \leq u \\ m \text { squarefree }}} \Pi\left(\mathbb{K}_{m}, x\right)
$$

where $\mathbb{K}_{m}=\mathbb{Q}(\sqrt{-m})$. We also put

$$
\sigma(x ; v, v)=\sigma(x ; v) .
$$

Clearly, the nonuniform bound (4) cannot be use to estimate $\sigma(x ; u, v)$, while (5) immediately implies that uniformly over $u$,

$$
\sigma(x ; u, v)=O\left(v x^{13 / 14} \log x\right) .
$$

Since we trivially have $\sigma(x ; u, v) \leq \pi(x)$, the above bound is nontrivial only for $v \leq x^{1 / 14}$. Here we obtain a more accurate bound which remains nontrivial for values of $v$ up to $x^{1 / 13-\varepsilon}$ for arbitrary $\varepsilon>0$ and sufficiently large $x$. 
Theorem 2. Suppose $\mathbf{E}$ does not have complex multiplication and also assume that the GRH holds. Then for $4 x \geq u>v \geq 1$ we have

$$
\sigma(x ; u, v) \leq(v x)^{55 / 59+o(1)}
$$

and

$$
\sigma(x ; v) \leq v^{13 / 14} x^{13 / 14+o(1)} .
$$

It is easy to check that the first bound of Theorem 2 is nontrivial and stronger than (6) in the range

$$
x^{3 / 56+\varepsilon} \leq v \leq x^{4 / 55-\varepsilon}
$$

for any fixed $\varepsilon>0$ and sufficiently large $x$.

Let

$$
\mathcal{M}(x)=\left\{m \in \mathbb{Z} \mid \Pi\left(\mathbb{K}_{m}, x\right)>0\right\}
$$

(where as before $\mathbb{K}_{m}=\mathbb{Q}(\sqrt{-m})$ ). An immediate implication of (5) is the bound

$$
\# \mathcal{M}(x) \gg \frac{x^{1 / 14}}{(\log x)^{2}} .
$$

see [1, Corollary 4]. We now observe that the first inequality of Theorem 2 implies that for almost all primes $p \leq x$ the corresponding Frobenius field is of discriminant at least $x^{1 / 13+o(1)}$. In particular, we have

$$
\max \mathcal{M}(x) \geq x^{1 / 13+o(1)} .
$$

\section{Character Sums}

For $p \nmid N$, we put

$$
a_{p}=p+1-\# \mathbf{E}_{p}\left(\mathbb{F}_{p}\right),
$$

where $\# \mathbf{E}_{p}\left(\mathbb{F}_{p}\right)$ is the number of $\mathbb{F}_{p}$-rational points of $\mathbf{E}_{p}$. When $p \mid N$, we simply put $a_{p}=1$. We recall that by the Hasse bound, $\left|a_{p}\right| \leq 2 p^{1 / 2}$, see [7].

We recall that the size of $\operatorname{III}_{p}$ is given by $\# \mathrm{III}_{p}=s_{p}^{2}$, where the integer $s_{p}$ is defined by the relation $4 p-a_{p}^{2}=s_{p}^{2} r_{p}$ with a squarefree integer $r_{p}$. Thus it is natural to use the square sieve [4] to study the distribution of \# $\mathrm{III}_{p}$. This requires nontrivial bounds of sums with the Jacobi symbols with $4 p-a_{p}^{2}$ 
modulo products $\ell_{1} \ell_{2}$ of two distinct primes. Accordingly, for an odd positive integer $n$ we define

$$
U(x ; n)=\sum_{p \leq x}\left(\frac{a_{p}^{2}-4 p}{n}\right),
$$

where, as usual, $(k / n)$ denotes the Jacobi symbol of $k$ modulo $n$.

The sum has been estimated by Cojocaru, Fouvry and Murty [3] and then sharpened by Cojocaru and Duke [2, Proposition 4.3]. Furthermore, when $n=\ell_{1} \ell_{2}$ is a product of two distinct primes, which is the only relevant case for this paper, Cojocaru and David [1, Theorem 3] give a stronger bound which we present here in the following form:

Lemma 3. Suppose $\mathbf{E}$ does not have complex multiplication and also assume that the GRH holds. Then for any real $x \geq 1$ and for any distinct primes $\ell_{1}, \ell_{2}>3$, we have

$$
U\left(x ; \ell_{1} \ell_{2}\right)=\frac{1}{\left(\ell_{1}^{2}-1\right)\left(\ell_{2}^{2}-1\right)} \pi(x)+O\left(\left(\ell_{1} \ell_{2}\right)^{3} x^{1 / 2} \log \left(\ell_{1} \ell_{2} x\right)\right) .
$$

We also need the following special case of the classical Burgess bound, see [6, Theorems 12.5] taken with $r=2$.

Lemma 4. For any real $u \geq v \geq 1$ and an odd square-free integer $s$,

$$
\sum_{u-v \leq m \leq u}\left(\frac{m}{s}\right) \ll v^{1 / 2} s^{3 / 16+o(1)} .
$$

As we have mentioned, a part of our improvement of (2) and (3) comes from bringing into the argument of [2] the following result of Heath-Brown [5].

Lemma 5. For any real positive $X$ and $Y$ with $X Y \rightarrow \infty$ and complex-valued function $f(m)$,

$$
\sum_{\substack{s \leq Y \\ s \text { odd squarefree }}}\left|\sum_{\substack{m \leq X \\ m}} f(m)\left(\frac{m}{s}\right)\right|^{2} \leq(X Y)^{o(1)}(X+Y) \sum_{1 \leq m \leq X}|f(m)|^{2} .
$$




\section{Square Multiples and Divisors of $4 p-a_{p}^{2}$}

As in [2], we define

$$
S_{m}(x)=\#\left\{p \leq x \mid m\left(4 p-a_{p}^{2}\right) \text { is a square }\right\} .
$$

Lemma 6. Suppose $\mathbf{E}$ does not have complex multiplication and also assume that the GRH holds. Then for any real $4 x \geq u \geq v \geq 1$, we have

$$
\sum_{\substack{u-v \leq m \leq u \\ m \text { squarefree }}} S_{m}(x) \leq v^{55 / 59} x^{55 / 59+o(1)} .
$$

Proof. Fix some

$$
z \geq(\log u)^{2}
$$

and assume that $x$ is sufficiently large. Then by [2, Bound (37)] we have

$$
S_{m}(x) \ll \frac{1}{\pi(z)^{2}} \sum_{n \leq 4 x^{2}} w_{m}(n)\left(\sum_{z \leq \ell \leq 2 z}\left(\frac{n}{\ell}\right)\right)^{2},
$$

where the inner sum is taken over all primes $\ell \in[z, 2 z]$ and

$$
w_{m}(n)=\#\left\{p \leq x \mid m\left(4 p-a_{p}^{2}\right)=n\right\} .
$$

We now derive

$$
\begin{aligned}
\sum_{\substack{u-v \leq m \leq u \\
m \text { squarefree }}} S_{m}(x) & \leq \sum_{u-v \leq m \leq u} S_{m}(x) \\
& \leq \frac{1}{\pi(z)^{2}} \sum_{u-v \leq m \leq u} \sum_{n \leq 4 x^{2}} w_{m}(n)\left(\sum_{z \leq \ell \leq 2 z}\left(\frac{n}{\ell}\right)\right)^{2} \\
& =\frac{1}{\pi(z)^{2}} \sum_{u-v \leq m \leq u} \sum_{z \leq \ell_{1}, \ell_{2} \leq 2} \sum_{n \leq 4 x^{2}} w_{m}(n)\left(\frac{n}{\ell_{1} \ell_{2}}\right) .
\end{aligned}
$$

Separating $\pi(z)$ diagonal terms with $\ell_{1}=\ell_{2}$, we obtain

$$
\sum_{u-v \leq m \leq u} S_{m}(x) \ll \frac{1}{\pi(z)} \Sigma_{1}+\frac{1}{\pi(z)^{2}} \Sigma_{2},
$$


where

$$
\begin{aligned}
\Sigma_{1} & =\sum_{u-v \leq m \leq u} \sum_{n \leq 4 x^{2}} w_{m}(n), \\
\Sigma_{2} & =\sum_{u-v \leq m \leq u} \sum_{n \leq 4 x^{2}} \sum_{z \leq \ell_{1}<\ell_{2} \leq 2 z} w_{m}(n)\left(\frac{n}{\ell_{1} \ell_{2}}\right) .
\end{aligned}
$$

We estimate the first sums trivially as

$$
\Sigma_{1} \leq \sum_{u-v \leq m \leq u} \pi(x) \leq v \pi(x) .
$$

For the second sum, we note that

$$
\begin{aligned}
\sum_{n \leq 4 x^{2}} w_{m}(n)\left(\frac{n}{\ell_{1} \ell_{2}}\right) & =\sum_{p \leq x}\left(\frac{m\left(4 p-a_{p}^{2}\right)}{\ell_{1} \ell_{2}}\right) \\
& =\left(\frac{-m}{\ell_{1} \ell_{2}}\right) \sum_{p \leq x}\left(\frac{a_{p}^{2}-4 p}{\ell_{1} \ell_{2}}\right)=\left(\frac{-m}{\ell_{1} \ell_{2}}\right) U\left(x ; \ell_{1} \ell_{2}\right) .
\end{aligned}
$$

Thus, changing the order of summation, we derive

$$
\Sigma_{2}=\sum_{z \leq \ell_{1}<\ell_{2} \leq 2 z} \sum_{u-v \leq m \leq u}\left(\frac{-m}{\ell_{1} \ell_{2}}\right) U\left(x ; \ell_{1} \ell_{2}\right) .
$$

By Lemma 3, we have

$$
U\left(x ; \ell_{1} \ell_{2}\right) \ll x^{1+o(1)}\left(\ell_{1} \ell_{2}\right)^{-2}+x^{1 / 2+o(1)}\left(\ell_{1} \ell_{2}\right)^{3}=x^{1+o(1)} z^{-4}+x^{1 / 2+o(1)} z^{6},
$$

which yields the the estimate

$$
\Sigma_{2} \leq\left(x^{1+o(1)} z^{-4}+x^{1 / 2+o(1)} z^{6}\right) \sum_{z \leq \ell_{1}<\ell_{2} \leq 2 z}\left|\sum_{u-v \leq m \leq u}\left(\frac{m}{\ell_{1} \ell_{2}}\right)\right| .
$$

We now apply Lemma 4 to derive from (11) that

$$
\Sigma_{2} \leq x^{o(1)}\left(x z^{-4}+x^{1 / 2} z^{6}\right) v^{1 / 2} z^{19 / 8} .
$$

Substitution of (10) and (12) in (9) leads us to the bound:

$$
\begin{aligned}
\sum_{\substack{u-v \leq m \leq u \\
m \text { squarefree }}} S_{m}(x) & \leq x^{o(1)}\left(x v z^{-1}+v^{1 / 2} x z^{-29 / 8}+v^{1 / 2} x^{1 / 2} z^{51 / 8}\right) \\
& \leq x^{o(1)}\left(v x z^{-1}+v^{1 / 2} x^{1 / 2} z^{51 / 8}\right) .
\end{aligned}
$$


Choosing

$$
z=(v x)^{4 / 59}
$$

(thus (7) holds), we conclude the proof.

For any fixed $\varepsilon>0$, Lemma 6 gives a nontrivial estimate provided that $v \leq x^{4 / 55-\varepsilon}$ uniformly over $u$.

In the case of $u=v$, we now obtain a slightly better bound.

Lemma 7. Suppose $\mathbf{E}$ does not have complex multiplication and also assume that the GRH holds. Then for any real $4 x \geq u \geq 1$, we have

$$
\sum_{\substack{1 \leq m \leq v \\ m \text { squarefree }}} S_{m}(x) \leq v^{13 / 14} x^{13 / 14+o(1)}
$$

Proof. We proceed as in the proof of Lemma 6, however, we always preserve the condition that $m$ is square-free. Then we can estimate $\Sigma_{2}$ by using Lemma 5 instead of Lemma 4 .

More precisely, applying the Cauchy inequality and then using Lemma 5 with $X=v, Y=4 z^{2}$ and $f(m)=1$, we obtain

$$
\begin{aligned}
\sum_{z \leq \ell_{1}<\ell_{2} \leq 2 z}\left|\sum_{\substack{1 \leq m \leq v \\
m}}\left(\frac{m}{\ell_{1} \ell_{2}}\right)\right| \ll\left(z^{2} \sum_{z \leq \ell_{1}<\ell_{2} \leq 2 z}\left|\sum_{\substack{1 \leq m \leq v \\
\text { squarefree }}}\left(\frac{m}{\ell_{1} \ell_{2}}\right)\right|^{2}\right)^{1 / 2} \\
\leq\left(x^{o(1)} v z^{2}\left(v+z^{2}\right)\right)^{1 / 2}=x^{o(1)}\left(v z+v^{1 / 2} z^{2}\right) .
\end{aligned}
$$

We now derive from (11) that

$$
\Sigma_{2} \leq x^{o(1)}\left(v x z^{-3}+v^{1 / 2} x z^{-2}+x^{1 / 2} v z^{7}+v^{1 / 2} x^{1 / 2} z^{8}\right) .
$$

Substitution of (10) and (13) in (9) leads us to the bound:

$$
\sum_{\substack{1 \leq m \leq v \\ m \text { squarefree }}} S_{m}(x) \leq x^{o(1)}\left(v x z^{-1}+v x z^{-5}+v^{1 / 2} x z^{-4}+v x^{1 / 2} z^{5}+v^{1 / 2} x^{1 / 2} z^{6}\right) .
$$

Clearly the second and the third terms are both dominated by the first term. Hence the bound simplifies as

$$
\sum_{\substack{1 \leq m \leq v \\ m \text { squarefree }}} S_{m}(x) \leq x^{o(1)}\left(v x z^{-1}+v x^{1 / 2} z^{5}+v^{1 / 2} x^{1 / 2} z^{6}\right) .
$$


If we choose

$$
z=(v x)^{1 / 14}
$$

(thus (77) holds), to balance the first and the third terms as $(v x)^{13 / 14}$, which also gives $v^{19 / 14} x^{6 / 7}$ for the second term, we obtain

$$
\sum_{\substack{1 \leq m \leq v \\ m \text { squarefree }}} S_{m}(x) \leq x^{o(1)}\left((v x)^{13 / 14}+v^{12 / 7} x^{17 / 14}\right) .
$$

Clearly the bound is nontrivial only if $(v x)^{13 / 14} \leq x$ or $v \leq x^{1 / 13}$ in which case $(v x)^{13 / 14}>v^{19 / 14} x^{6 / 7}$, thus the first term always dominates.

Also as in [2], we define

$$
D(x, y)=\sum_{y \leq n \leq 2 x^{1 / 2}} \pi_{n}(x),
$$

where

$$
\pi_{n}(x)=\#\left\{p \leq x\left|p \nmid N, n^{2}\right| \# \mathrm{III}_{p}\right\} .
$$

This function is of independent interest. Our next result improves [2, Proposition 5.2].

Lemma 8. Suppose $\mathbf{E}$ does not have complex multiplication and also assume that the GRH holds. Then for any real $1 \leq y \leq 2 x^{1 / 2}$, we have

$$
D(x, y) \leq \begin{cases}x^{29 / 16+o(1)} y^{-7 / 4}, & \text { if } y<x^{5 / 12}, \\ x^{13 / 7+o(1)} y^{-13 / 7}, & \text { otherwise. }\end{cases}
$$

Proof. It is easy to check that [2, Bound (36)] can in fact be replaced by the following estimate

$$
D(x, y) \leq x^{o(1)} \sum_{\substack{m \leq 4 x / y^{2} \\ m \text { squarefree }}} S_{m}(x)
$$

We note that this bound differs from [2, Bound (36)] only in that we still require $m$ to be squarefree. This condition is present in all considerations which have lead to [2, Bound (36)], but is not included in that bound. Preserving this condition does not give any advantage for the argument of [2] but is important for us. Using Lemma 6 for $y<x^{5 / 12}$ and Lemma 7 otherwise, we obtain the result. 


\section{Proofs of Theorems 1 and 2}

As in the proof of [2, Proposition 5.3] we see that for any $1 \leq y \leq 2 x^{1 / 2}$ we have

$$
\pi_{T S}(x)=\alpha \pi(x)+O\left(D(x, y)+x^{1 / 2+o(1)} y\right)
$$

where $\alpha$ is as in (11). Using the second bound of Lemma 8 , we derive

$$
\pi_{T S}(x)=\alpha \pi(x)+O\left(x^{13 / 7+o(1)} y^{-13 / 7}+x^{1 / 2+o(1)} y\right),
$$

and then selecting $y=x^{19 / 40}$, we conclude the proof of Theorem 11.

To proof Theorem 2, as in [1], we note that

$$
\sigma(x ; u, v) \leq \sum_{\substack{u-v \leq m \leq u \\ m \text { squarefree }}} S_{m}(x) .
$$

Now Lemmas 6 and 7 imply the result.

\section{$5 \quad$ Remarks}

Under some additional assumptions, Cojocaru and David [1, Theorem 3] give sharper bounds on the error term in the asymptotic formula of Lemma 3. In turn, this leads to further sharpening the bound of Theorem 1 (under the same additional assumptions).

We also note that, Lemma 8 shows that the bound $\# \mathrm{III}_{p} \leq x^{12 / 13+o(1)}$ holds for all but $o(\pi(x))$ primes $p \leq x$.

It would be very interesting to obtain an unconditional proof of the asymptotic formula (1) with $R(x)=o(\pi(x))$. For this one needs a nontrivial estimate on $D(x, y)$ for rather small values of $y$. Although Lemma 8 reduces the gap, it still remains quite wide. Although it is possible to obtain an unconditional version of Lemma 3 it seems to be too weak to leads to an asymptotic formula for $\pi_{T S}(x)$. However most certainly it can be used to show that the bound $\# \mathrm{III}_{p}=o(x)$ (or even a stronger and more explicit bound) holds for all but $o(\pi(x))$ primes $p \leq x$.

\section{Acknowledgement}

The author is grateful to Alina Cojocaru, Bjorn Poonen and Joseph Silverman for useful comments. 
This work was supported in part by ARC grant DP0556431.

\section{References}

[1] A. C. Cojocaru and C. David, 'Frobenius fields for elliptic curves', Amer. J. Math., (to appear).

[2] A. C. Cojocaru and W. Duke, 'Reductions of an elliptic curve and their Tate-Shafarevich groups', Math. Ann., 329 (2004), 513-534.

[3] A. C. Cojocaru, É. Fouvry and M. R. Murty, 'The square sieve and the Lang-Trotter conjecture', Canadian J. Math., 57 (2005), 1155-1177.

[4] D. R. Heath-Brown, 'The square sieve and consecutive squarefree numbers', Math. Ann., 266 (1984), 251-259.

[5] D. R. Heath-Brown, 'A mean value estimate for real character sums', Acta Arith., 72 (1995), 235-275.

[6] H. Iwaniec and E. Kowalski, Analytic number theory, Amer. Math. Soc., Providence, RI, 2004.

[7] J. H. Silverman, The arithmetic of elliptic curves, Springer-Verlag, Berlin, 1995. 